\title{
Interações Medicamentosas na Farmacoterapia de Idosos com Câncer atendidos em um Ambulatório de Onco-Hematologia
}

\author{
Drug Interactions in Elderly Cancer Patients Treated at a Hematology-Oncology Outpatient Clinic \\ Interacciones Farmacológicas en la Farmacoterapia de Adultos Mayores con Cáncer Atendidos en un Ambulatorio \\ de Onco-Hematología
}

Caroline de Oliveira Faria '; Cristiane Moreira Reis²; Andrezza Gouvêa Santos ${ }^{3}$; Adriano Max Moreira Reis ${ }^{4}$

Resumo

Introdução: Mais de 50\% dos diagnósticos de câncer ocorrem na população idosa. Esse grupo etário possui outros problemas de saúde concomitantes à neoplasia que aumentam o risco de polifarmácia e interaçóes medicamentosas. Objetivo: Identificar a frequência das interaçôes medicamentosas potenciais e analisar os fatores associados na farmacoterapia de idosos com câncer. Método: Estudo transversal realizado em um ambulatório de onco-hematologia de um hospital de ensino. Foram entrevistados 160 idosos submetidos à terapia antineoplásica parenteral. Registraram-se informaçóes sobre farmacoterapia, diagnóstico da neoplasia, outros problemas de saúde e funcionalidade. A funcionalidade foi determinada usando o Vulnerable Elders Survey. As informaçóes clínicas foram coletadas em prontuário. Identificaram-se interaçôes medicamentosas potenciais empregando o software Drug Interaction Checking. A regressão logística foi utilizada para determinar os fatores associados às interaçóes medicamentosas potenciais. Resultados: Noventa e sete (60,6\%) idosos apresentaram pelo menos uma interação medicamentosa e 42 (26,3\%), interação envolvendo um medicamento antineoplásico. Ciclofosfamida e fluouracila foram os mais envolvidos em interações. O mecanismo farmacodinâmico foi responsável por $52,5 \%$ das interaçóes. Identificou-se que $51 \%$ das interaçôes foram classificadas como graves. A regressão logística mostrou que interaçóes medicamentosas estiveram associadas de forma independente com número de medicamentos [OR=1,51; IC95\% $(1,277-1,78), \mathrm{p}<0,0001)$ e de problemas de saúde [OR=1,39; IC95\% (1,030-1,880), p=0,031]. Conclusáo: A frequência de interaçóes medicamentosas potenciais em idosos com câncer em terapia antineoplásica parenteral foi elevada, ainda que a proporção de interaçôes graves corresponda à aproximadamente metade do total de interaçôes. O número de problemas de saúde e o de medicamentos apresentaram associação positiva com interaçốes medicamentosas.

Palavras-chave: Interações de Medicamentos; Idosos; Antineoplásicos; Tratamento Farmacológico; Neoplasia.

\section{Abstract}

Introduction: More than $50 \%$ of all cancer diagnoses occur in the elderly population. This age group has other health problems concomitant with neoplasia, a scenario that increases the likelihood of polypharmacy and the risk of drug interactions. Purpose: To identify the frequency of and factors associated with drug interactions in the pharmacological treatment of elderly cancer patients. Method: This was a cross-sectional study conducted at the hematology-oncology outpatient clinic of a university hospital. We interviewed 160 elderly patients receiving intravenous antineoplastic therapy, collecting information about antineoplastic and other pharmacotherapy, the diagnosis of neoplasia, other health problems, and functionality. Functionality was assessed with the Vulnerable Elders Survey. Clinical data were collected from the patient charts. To identify potential drug interactions, we used drug interaction checking software. Logistic regression was used in order to identify factors associated with drug interactions. Results: Of the 160 elderly patients interviewed, 97 $(60.6 \%)$ presented at least one drug interaction and $42(26.3 \%)$ presented at least one interaction with an antineoplastic drug. Cyclophosphamide and fluorouracil were the drugs most often involved in interactions. Of the interactions, $52.5 \%$ were attributable to a pharmacodynamic mechanism of action and $51.0 \%$ were classified as severe. Logistic regression showed that drug interactions were independently associated with the number of drugs taken $(\mathrm{OR}=1.51,95 \% \mathrm{CI}: 1.277-1.78, \mathrm{p}<0.0001)$ and with the number of health problems (OR=1.39, $95 \%$ CI: $1.030-1.880, \mathrm{p}=0.031)$. Conclusion: The frequency of drug interactions in elderly cancer patients receiving intravenous antineoplastic therapy is high, and severe interactions account for approximately half of all such interactions. The number of health problems and the number of drugs taken appear to be positively associated with drug interactions.

Key words: Drug Interactions; Aged; Antineoplastic Agents; Drug Therapy; Neoplasms.

\section{Resumen}

Introducción: Más del 50\% de los diagnósticos de cáncer ocurren en la población anciana. Este grupo de edad posee otros problemas de salud concomitantes a la neoplasia que aumenta el riesgo de polifarmacia e interacciones medicamentosas. Objetivo: Identificar la frecuencia y los factores asociados con interacciones farmacológicas potenciales en la farmacoterapia de adultos mayores con cáncer. Método: Estudio transversal realizado en un ambulatorio de onco-hematología de un hospital universitario. Se entrevistó a 160 adultos mayores sometidos a la terapia antineoplásica parenteral. Se registró informaciones acerca de la farmacoterapia antineoplásica y no antineoplásica, diagnóstico de la neoplasia, otros problemas de salud y funcionalidad. La información clínica fue recolectada en los registros de los pacientes. La funcionalidad se determinó utilizando el Vulnerable Elders Survey. Se identificaron las interacciones medicamentosas potenciales empleando el programa Drug Interaction Checking y se utilizó la regresión logística para determinar los factores asociados a las interacciones farmacológicas. Resultados: Un total de 97 (60,6\%) de los ancianos presentó al menos una interacción farmacológica y $42(26,3 \%)$ presentaron interacción envolviendo un medicamento antineoplásico. Los antineoplásicos ciclofosfamida y fluouracilo fueron los más implicados en las interacciones. El mecanismo farmacodinámico fue responsable por $52,5 \%$ de las interacciones. Se identificó que $51 \%$ de las interacciones fueron clasificadas como graves. La regresión logística mostró que las interacciones medicamentosas se asociaron de forma independiente con el número de medicamentos [OR=1,51; IC95\% $(1,277$ $1,78), \mathrm{p}<0,0001)$ y el número de problemas de salud $[\mathrm{OR}=1,39$; IC $95 \%(1,030$ 1,880), $\mathrm{p}=0,031]$. Conclusiones: La frecuencia de interacciones farmacológicas potenciales en ancianos con cáncer sometidos a terapia antineoplásica parenteral fue elevada aunque la proporción de interacciones graves corresponde a aproximadamente la mitad del total de interacciones. El número de problemas de salud y el número de medicamentos presentaron una asociación positiva con las interacciones farmacológicas.

Palabras clave: Interacciones Medicamentosas; Ancianos; Antineoplásicos; Tratamiento Farmacológico; Neoplasia.

${ }^{1}$ Farmacêutica. Especialista em Saúde do Idoso. Programa de Residência Multiprofissional em Saúde do Idoso do Hospital das Clínicas. Universidade Federal de Minas Gerais (UFMG). Belo Horizonte (MG), Brasil. E-mail: carol.ofaria@hotmail.com.

${ }^{2}$ Mestre em Assistência Farmacêutica e Medicamentos pela UFMG. Belo Horizonte (MG), Brasil. E-mail: chrismestry@yahoo.com.br.

${ }^{3}$ Farmacêutica-Residente do Hospital das Clínicas. UFMG. Belo Horizonte (MG), Brasil.E-mail: gouvea.andrezza@gmail.com.

${ }^{4}$ PhD. Faculdade de Farmácia. UFMG. Belo Horizonte (MG), Brasil. E-mail: amreis@outlook.com

Endereço para correspondência: Caroline de Oliveira Faria. Av. Professor Alfredo Balena, 110 - Santa Efigênia. Belo Horizonte (MG), Brasil. CEP 30.130-100. 


\section{INTRODUÇÃO}

As melhorias nas condiçôes de saúde, ainda que de forma desigual nos diversos contextos sociais, proporcionaram aos indivíduos o aumento no número de anos vividos. Entretanto, associado à longevidade, há incremento do risco de doenças crônico-degenerativas; entre elas, as neoplasias ${ }^{1}$.

Nesse contexto, o número crescente de idosos acometidos por neoplasias associado ao envelhecimento populacional configura uma realidade no mundo todo. Mais de $50 \%$ dos diagnósticos de câncer e aproximadamente $70 \%$ dos óbitos por câncer ocorrem na população geriátrica² ${ }^{2}$.

Os idosos possuem outros problemas de saúde concomitantes à neoplasia, o que pode reduzir a tolerância ao tratamento antineoplásico, limitar indicaçóes terapêuticas e piorar o prognóstico. Os problemas de saúde também contribuem para o uso de múltiplos medicamentos o que, por sua vez, aumenta o risco de interaçóes medicamentosas e de eventos adversos relacionados aos medicamentos ${ }^{3}$.

$\mathrm{Na}$ população geriátrica em geral - mais vulnerável à ocorrência de eventos adversos a medicamentos - a prevalência e a significância clínica das interaçôes medicamentosas estâo bem documentadas ${ }^{3,4}$. Quanto à populaçâao específica de idosos com câncer, o número de investigaçóes internacionais vem crescendo e ganhando notoriedade ${ }^{4}$. Inclusive, as diretrizes do National Comprehensive Cancer Network para idosos com câncer recomendam a investigação e manejo de interaçóes medicamentosas 5 . Não obstante, na população de idosos brasileiros em uso de terapia antineoplásica, os estudos sobre interaçôes medicamentosas são escassos.

Diante do exposto, este trabalho justifica-se pela necessidade de investigações mais específicas relacionadas a esse grupo de idosos, a fim de orientar o manejo clínico e estratégias de prevenção de eventos adversos advindos de interaçôes medicamentosas.

O objetivo deste estudo foi identificar a frequência de interaçóes medicamentosas potenciais na farmacoterapia de idosos com câncer e analisar os fatores associados.

\section{MÉTODO}

Estudo transversal realizado no ambulatório de onco-hematologia de um hospital de ensino de Belo Horizonte, Minas Gerais, no período de julho a dezembro de 2015. A amostra de conveniência foi constituída de 160 idosos com diagnóstico de câncer que assinaram o Termo de Consentimento Livre Esclarecido. A investigação foi aprovada pelo Comitê de Ética em Pesquisa da
Universidade Federal de Minas Gerais, sob número CAAE 40511915.8.0000.5149.

Os critérios de inclusão no estudo foram: idade $\geq 60$ anos, com diagnóstico de neoplasia, em tratamento com medicamentos por via parenteral, classificados como: L01- Agentes Antineoplásicos ou L02-Terapia Endócrina, segundo a sistemática da Classificação Anatômica Terapêutica Clínica da Organização Mundial da Saúde (ATC/OMS), em uso de dois ou mais medicamentos, independente da classe terapêutica.

Os idosos e/ou acompanhantes foram entrevistados durante o período em que os medicamentos antineoplásicos ou de suporte estavam sendo infundidos ou durante o tempo em que os pacientes estavam aguardando atendimento para início da sessão de quimioterapia. Durante a entrevista, foram coletadas informaçóes sociodemográficas e farmacoterapêuticas. As informaçóes clínicas da neoplasia e dos demais problemas de saúde, bem como sobre o tratamento antineoplásico, foram coletadas no prontuário do paciente.

A variável dependente da investigação é a ocorrência de interaçôes medicamentosas potenciais. Essas foram identificadas empregando o software Drug Interaction Checking da IBM Micromedex ${ }^{\circ 6}$, aplicativo que possui bom desempenho na identificação de interaçóes medicamentosas em oncologia ${ }^{4}$. Interaçôes medicamentosas envolvendo associação de dois antineoplásicos não foram consideradas quando estes faziam parte do mesmo protocolo de tratamento. As interaçóes foram classificadas quanto ao mecanismo de ação, à gravidade e ao nível de evidência científica, segundo os critérios definidos nas monografias das interaçôes medicamentosas constantes do Drug Interaction Checking. O mecanismo de ação foi classificado em farmacocinético, farmacodinâmico, misto (farmacocinético e farmacodinâmico) e desconhecido. Foram analisadas apenas as interaçóes medicamentosas classificadas em: 1. Contraindicadas, quando os medicamentos náo são recomendados para uso concomitante; 2. Graves, quando a interação pode representar perigo à vida e/ou requerer intervençâo médica para diminuir ou evitar eventos adversos importantes; 3. Moderadas, que podem resultar em exacerbaçáo do problema de saúde do paciente e/ou requerer uma alteração no tratamento. Quanto ao nível de evidência científica, as interações foram classificadas em excelente, bom e razoável ${ }^{6}$.

As variáveis independentes foram referentes à caracterização sociodemográfica do paciente (sexo, idade, estado civil), às características clínicas (Classificação Estatística Internacional de Doenças e Problemas Relacionados com a Saúde - CID 10 da neoplasia, tipo da neoplasia, problemas de saúde, vulnerabilidade) e 
da farmacoterapia (terapia antineoplásica, terapia não antineoplásica, número de medicamentos utilizados). Os medicamentos foram classificados segundo o subgrupo químico (nível 4) da sistemática ATC/OMS. A vulnerabilidade do idoso foi avaliada por meio do instrumento Vulnerable Elders Survey-13 (VES-13), adaptado transculturalmente para o Brasil ${ }^{7}$. Indivíduos cujas pontuaçóes são $\geq 3$ apresentam risco 4,2 vezes maior de declínio funcional e morte em dois anos, quando comparados aos indivíduos com escores menores. A versão brasileira do VES-13 apresentou propriedades psicométricas consistentes em uma amostra de idosos brasileiros com câncer? .

Houve dupla digitaçáo do banco de dados construído no programa EpiData versão 3.1. A análise descritiva dos dados foi realizada determinando as frequências e porcentagens para as características das variáveis categóricas, medidas de tendência central (média e mediana) e medidas de dispersão (desvio-padrão e intervalo interquartil - IQR) para as variáveis quantitativas. As variáveis categóricas foram comparadas com a variável dependente utilizando o teste Qui-Quadrado. $\mathrm{Na}$ análise univariada, foi utilizado o teste $t$-Student para variáveis quantitativas com distribuição normal e teste de Mann-Whitney para variáveis que não atenderam às suposiçóes de normalidade. O intervalo de confiança (IC) utilizado foi de $95 \%$ e o nível de significância $p<0,05$. As variáveis independentes com valores de $\mathrm{p}<0,25$ na análise univariada foram incluídas em modelo de regressão logística. A magnitude da associação entre as variáveis foi estimada utilizando odds ratio (OR) com IC igual a 95\% em ambos modelos: univariado e multivariado. A adequação do modelo final foi avaliada pelo Teste de Hosmer-Lemeshow. O IBM SPSS Statistics software package, versão 24.0 foi empregado para a realização das análises estatísticas.

\section{RESULTADOS}

A amostra - constituída em sua maioria por mulheres (57,5\%) - apresentou mediana de idade de 67,5 anos e IQR igual a 10. O estado civil casado foi declarado por $63,8 \%$ dos idosos. Os tumores sólidos constituíram o tipo mais comum de neoplasia $(97,6 \%)$ em comparação às neoplasias hematológicas (2,4\%). Quanto aos tumores sólidos, as classificaçôes mais frequentes foram: mama (28,1\%); colorretal (22,5\%); pulmão (7,5\%); estômago $(6,9 \%)$, próstata $(6,3 \%)$ e esôfago $(4,4 \%)$. Mieloma $(1,2 \%)$, linfoma $(0,6 \%)$ e leucemia $(0,65 \%)$ foram os subtipos de neoplasias hematológicas encontradas. Os problemas de saúde autodeclarados mais comuns foram: hipertensão arterial sistêmica $(33,9 \%)$; diabetes $(13,1 \%)$; artrite/artrose (10,6\%); depressão (10,2\%); e doenças da tireoide (5,9\%). A mediana no escore VES-13 que mensura a vulnerabilidade do idoso foi 1,5 IQR igual a cinco (Tabela 1).

Foram identificadas interações medicamentosas na farmacoterapia de $60,6 \%$ dos idosos; $26,3 \%$ apresentaram interações medicamentosas envolvendo antineoplásicos, sendo o número máximo de interaçôes envolvendo medicamentos antineoplásicos por idoso igual a cinco. Em relaçáo aos medicamentos para tratamento de doenças não neoplásicas, 52,5\% dos idosos apresentaram interaçôes medicamentosas e número máximo de 17 interaçōes por paciente envolvendo esses medicamentos (Tabela 1).

A Tabela 2 sumariza as interaçóes fármaco-fármaco envolvendo antineoplásicos, seus efeitos clínicos e frequências. É possível observar que os medicamentos antineoplásicos com maior frequência de interaçóes foram ciclofosfamida e fluoruracila, presentes em 35\% e $23 \%$, respectivamente, das interaçóes identificadas. Foram observados 18 tipos de interaçóes. As interaçóes classificadas como graves corresponderam à metade do total de interaçóes encontradas. A outra metade foi classificada como interação do tipo moderada. Quanto ao mecanismo de ação envolvido, mais da metade das interaçóes $(55,6 \%)$ compartilhavam o tipo farmacocinético. Em torno de $22 \%$ das interaçôes envolviam mecanismos desconhecidos, 16,7\% mecanismos farmacodinâmicos e 5,6\% mecanismos mistos. O nível de evidência científica foi considerado razoável para a maioria das interaçóes e classificado como bom e excelente em aproximadamente $38,9 \%$ e 5,6\% das interaçóes, respectivamente.

As interaçóes fármaco-fármaco mais frequentes envolvendo medicamentos para tratamento de doenças não neoplásicas podem ser observadas na Tabela 3. No total, 118 tipos de interaçôes foram identificados. Quanto à gravidade, aproximadamente $51 \%$ das interaçóes foram classificadas como graves e $49 \%$ moderadas. O mecanismo de ação farmacodinâmico foi o mais frequente e responsável por $52,5 \%$ das interaçóes. Para $23,7 \%$ das interaçôes, o mecanismo envolvido era farmacocinético, $17,8 \%$ eram mecanismos desconhecidos e $5,9 \%$ eram mecanismos mistos. O nível de evidência científica foi considerado razoável para $58,5 \%$ das interaçôes e classificado como bom e excelente em $29,7 \%$ e $11,9 \%$ das interaçôes, respectivamente.

$\mathrm{Na}$ análise univariada pelo teste Qui-Quadrado, verificou-se associação com interaçóes medicamentosas com $\mathrm{p}$ valor $<0,25$ para as seguintes variáveis com respectivos OR e IC 95\%: idade maior que 70 anos [OR=1,76; IC 95\% (0,90-3,46), $\mathrm{p}=0,095]$; depressão [OR=2,39; IC 95\% (1,00-5,69), $\mathrm{p}=0,046]$; hipertensão arterial sistêmica [OR 2,3; IC 95\% $(1,15-4,50)$ p=0,160]; 
Tabela 1. Características sociodemográficas e clínicas da amostra (160 idosos)

\begin{tabular}{|c|c|c|}
\hline Características & \multicolumn{2}{|c|}{ Valores } \\
\hline Idade em anos [mediana (amplitude interquartil)] & 67,5 & $(63-73)$ \\
\hline Sexo [feminino $n(\%)]$ & 92 & $(57,5)$ \\
\hline Estado civil [casado n (\%)] & 102 & $(63,8)$ \\
\hline Escore VES-13* [mediana (amplitude interquartil)] & 1,5 & $(0-5)$ \\
\hline \multicolumn{3}{|l|}{ Tipos de neoplasias - n (\%) } \\
\hline \multicolumn{3}{|l|}{ Tumores sólidos } \\
\hline Mama & 45 & $(28,1)$ \\
\hline Colorretal & 36 & $(22,5)$ \\
\hline Pulmão & 12 & $(7,5)$ \\
\hline Estômago & 11 & $(6,9)$ \\
\hline Próstata & 10 & $(6,3)$ \\
\hline Esôfago & 7 & $(4,4)$ \\
\hline Outros & 35 & $(21,9)$ \\
\hline \multicolumn{3}{|l|}{ Neoplasias hematológicas } \\
\hline Mielomas & 2 & $(1,2)$ \\
\hline Linfomas & 1 & $(0,6)$ \\
\hline Leucemias & 1 & $(0,6)$ \\
\hline Número de problemas de saúde [mediana (amplitude interquartil)] & 2,0 & $(1,0-3,0)$ \\
\hline \multicolumn{3}{|l|}{ Problemas de saúde - n (\%) } \\
\hline Hipertensão arterial sistêmica & 109 & $(33,9)$ \\
\hline Diabetes & 42 & $(13,1)$ \\
\hline Artrite/artrose & 34 & $(10,6)$ \\
\hline Depressão & 33 & $(10,2)$ \\
\hline Doenças da tireoide & 19 & $(5,9)$ \\
\hline Outros & 85 & $(26,3)$ \\
\hline \multicolumn{3}{|l|}{ Farmacoterapia } \\
\hline $\begin{array}{l}\text { Número de medicamentos utilizados para tratamento de doenças não neoplásicas e } \\
\text { neoplásicas por paciente [mediana (amplitude interquartil)] }\end{array}$ & 9 & $(7-11)$ \\
\hline Pacientes com interações medicamentosas - n (\%) & 97 & $(60,6)$ \\
\hline Pacientes com interações envolvendo antineoplásicos parenterais - n (\%) & 42 & $(26,3)$ \\
\hline $\begin{array}{l}\text { Pacientes com interações envolvendo medicamentos para tratamento de doenças } \\
\text { não neoplásicas - n (\%) }\end{array}$ & 84 & $(52,5)$ \\
\hline Número máximo de interações por paciente & 17 & \\
\hline Número máximo de interações por paciente envolvendo antineoplásicos parenterais & 5 & \\
\hline
\end{tabular}

* VES-13 - Vulnerable Elders Survey-13: indivíduos cujas pontuaçốes são $\geq 3$ apresentam risco 4,2 vezes maior de declínio funcional e morte em dois anos, quando comparados aos indivíduos com escores menores.

diabetes [OR 1,64; IC 95\% (0,77-3,47) p=0,193]; e artrite/artrose [OR 4,94; IC 95\% $(1,80-13,60) \mathrm{p}=0,001$ ]. Foi encontrada associação significativa entre as interaçôes medicamentosas e o número de medicamentos $(\mathrm{p}<0,0001)$, e o número de problemas de saúde ( $\mathrm{p}<0,0001)$, segundo o Teste U Mann-Whitney. As variáveis categóricas com $\mathrm{p}$ valor $<0,25$ e as quantitativas com associação significativa com interaçôes foram incluídas no modelo de regressão logística multivariada. Apenas as variáveis número de medicamentos $[\mathrm{OR}=1,51$; IC 95\% (1,277-1,78), $\mathrm{p}<$ $0,0001)$ e número de problemas de saúde $[\mathrm{OR}=1,39$; IC $95 \%(1,030-1,880), \mathrm{p}=0,031]$ permaneceram independentemente associadas à ocorrência de interaçóes medicamentosas.

\section{DISCUSSÃO}

No presente estudo, a frequência de interaçóes fármaco-fármaco foi elevada, acometendo aproximadamente dois terços dos idosos. Estudos prévios envolvendo exclusivamente a população oncogeriátrica evidenciaram ampla variabilidade na frequência de interaçóes com resultados entre $16 \%$ e $75 \%{ }^{8-13}$. A metodologia utilizada, a heterogeneidade da população estudada, a sensibilidade e especificidade dos softwares para detecção de interaçôes justificam essa variabilidade ${ }^{14}$.

Destaca-se a prevalência - em torno de $50 \%$ - de interaçóes classificadas como graves; ou seja, que representam riscos de eventos adversos importantes e 
Tabela 2. Descrição das interações medicamentosas envolvendo antineoplásicos

\begin{tabular}{|c|c|c|c|c|c|c|}
\hline Interação & Efeito clínico & Gravidade & Mecanismo & $\begin{array}{l}\text { Nível de } \\
\text { evidência }\end{array}$ & $\begin{array}{l}\text { Frequência } \\
\text { absoluta }\end{array}$ & $\begin{array}{l}\text { Frequência } \\
\text { relativa }\end{array}$ \\
\hline Ciclofosfamida + ondansetrona & $\begin{array}{l}\text { Redução dos níveis séricos de } \\
\text { ciclofosfamida }\end{array}$ & Moderada & Desconhecido & Razoável & 16 & $22,9 \%$ \\
\hline Fluoruracila + hidroclorotiazida & $\begin{array}{l}\text { Aumento do risco de } \\
\text { mielossupressão }\end{array}$ & Moderada & Farmacocinético & Razoável & 13 & $18,6 \%$ \\
\hline Doxorrubicina + dexametasona & $\begin{array}{l}\text { Redução dos níveis séricos de } \\
\text { doxorrubicina }\end{array}$ & Grave & Misto & Razoável & 12 & $17,1 \%$ \\
\hline Paclitaxel + sinvastatina & $\begin{array}{l}\text { Alterações nos níveis séricos de } \\
\text { paclitaxel }\end{array}$ & Moderada & Farmacocinético & Razoável & 7 & $10,0 \%$ \\
\hline Ciclofosfamida + hidroclorotiazida & $\begin{array}{l}\text { Aumento dos níveis séricos de } \\
\text { ciclofosfamida e dos riscos de } \\
\text { mielosupressão }\end{array}$ & Grave & Farmacocinético & Bom & 5 & $7,1 \%$ \\
\hline Cisplatina + furosemida & $\begin{array}{l}\text { Aumento do risco de nefrotoxicidade } \\
\text { e ototoxicidade }\end{array}$ & Grave & Farmacodinâmico & Razoável & 3 & $4,3 \%$ \\
\hline Ciclosfosfamida + alopurinol & $\begin{array}{l}\text { Aumento do risco de } \\
\text { mielosupressão, náuseas e vômitos }\end{array}$ & Grave & Farmacocinético & Bom & 2 & $2,9 \%$ \\
\hline Fluoruracila + varfarina & Aumento do risco de sangramento & Grave & Farmacocinético & Excelente & 2 & $2,9 \%$ \\
\hline Ácido folínico + fenitoína & Redução da eficácia da fenitoína & Moderada & Desconhecido & Razoável & 1 & $1,4 \%$ \\
\hline Ciclofosfamida + glimepirida & Aumento do risco de hipoglicemia & Moderada & Desconhecido & Razoável & 1 & $1,4 \%$ \\
\hline Cisplatina + varfarina & $\begin{array}{l}\text { Aumento dos valores da razão } \\
\text { normatizada internacional e do risco } \\
\text { de sangramento }\end{array}$ & Moderada & Desconhecido & Bom & 1 & $1,4 \%$ \\
\hline Claritromicina + paclitaxel & $\begin{array}{l}\text { Aumento dos níveis séricos do } \\
\text { paclitaxel }\end{array}$ & Moderada & Farmacocinético & Razoável & 1 & $1,4 \%$ \\
\hline Dexametasona + talidomida & $\begin{array}{l}\text { Aumento do risco de necrólise } \\
\text { epidérmica tóxica }\end{array}$ & Grave & Farmacocinético & Bom & 1 & $1,4 \%$ \\
\hline Doxorrubicina + paroxetina & $\begin{array}{l}\text { Redução dos níveis séricos de } \\
\text { doxorrubicina }\end{array}$ & Moderada & Farmacocinético & Razoável & 1 & $1,4 \%$ \\
\hline Fluoruracila + fenitoína & $\begin{array}{l}\text { Aumento dos níveis séricos de } \\
\text { fenitoína e do risco de efeitos } \\
\text { adversos }\end{array}$ & Moderada & Farmacocinético & Bom & 1 & $1,4 \%$ \\
\hline Gencitabina + varfarina & Aumento do risco de sangramento & Grave & Farmacocinético & Bom & 1 & $1,4 \%$ \\
\hline Leuprolide + trazodona & $\begin{array}{l}\text { Aumento do risco de prolongamento } \\
\text { do intervalo QT }\end{array}$ & Grave & Farmacodinâmico & Razoável & 1 & $1,4 \%$ \\
\hline Leuprolide + venlafaxina & $\begin{array}{l}\text { Aumento do risco de prolongamento } \\
\text { do intervalo QT }\end{array}$ & Grave & Farmacodinâmico & Razoável & 1 & $1,4 \%$ \\
\hline Total & & & & & 70 & $100 \%$ \\
\hline
\end{tabular}

requerem intervenção clínica. Popa et.al ${ }^{10}$ investigaram a associaçáo entre interaçóes medicamentosas e quimiotoxicidade em idosos e reportaram risco de quimiotoxicidade não hematológica duas vezes maior a cada interação classificada como grave, e três vezes maior quando esta envolvia fármacos antineoplásicos. De acordo com os autores, esse achado ressalta a importância do rastreio contínuo de interaçôes, inclusive, antes do início do tratamento antineoplásico.

Entre as interações medicamentosas envolvendo antineoplásicos, ciclofosfamida e fluoruracila foram os mais prevalentes. A interação entre ciclofosfamida e antieméticos antagonistas do receptor da serotonina (como a ondansetrona) foi descrita em um trabalho revisional que orienta a escolha do antiemético, baseada na eficácia, segurança e no risco de interaçóes medicamentosas ${ }^{15}$.

Os fármacos ciclofosfamida e fluoruracila compartilham o mesmo desfecho clínico quando interagem com diuréticos tiazídicos, aumentando os riscos de eventos adversos, entre os quais, a mielossupressão apresenta-se como de maior gravidade. Estudos prévios também identificaram a ocorrência dessas interaçôes em pacientes oncológicos ${ }^{11,16-18}$. Na população geriátrica - cujo risco de mielossupressão é maior que em outras faixas etárias - as 
Tabela 3. Descrição das dez interações medicamentosas mais comuns envolvendo medicamentos para tratamento de doenças não neoplásicas

\begin{tabular}{|c|c|c|c|c|c|c|}
\hline Interação & Efeito clínico & Gravidade & Mecanismo & $\begin{array}{l}\text { Nível de } \\
\text { evidência }\end{array}$ & $\begin{array}{l}\text { Frequência } \\
\text { absoluta }\end{array}$ & $\begin{array}{l}\text { Frequência } \\
\text { relativa }\end{array}$ \\
\hline Ondansetrona + tramadol & $\begin{array}{l}\text { Aumento do risco de síndrome } \\
\text { serotoninérgica }\end{array}$ & Grave & Farmacodinâmico & Razoável & 14 & $6,0 \%$ \\
\hline AAS* + dexametasona & $\begin{array}{l}\text { Aumento do risco de úlcera } \\
\text { gastrointestinal } \\
\text { Redução dos níveis séricos do AAS }\end{array}$ & Moderada & Misto & Bom & 11 & $4,7 \%$ \\
\hline Anlodipino + sinvastatina & $\begin{array}{l}\text { Aumento dos níveis séricos de } \\
\text { sinvastatina e dos riscos de } \\
\text { miopatia, inclusive, de rabdomiólise }\end{array}$ & Grave & Farmacocinético & Bom & 8 & $3,4 \%$ \\
\hline Dexametasona + varfarina & $\begin{array}{l}\text { Aumento do risco de sangramento } \\
\text { Redução da atividade da varfarina }\end{array}$ & Moderada & Farmacocinético & Bom & 8 & $3,4 \%$ \\
\hline Amitriptlina + ondansetrona & $\begin{array}{l}\text { Aumento do risco de prolongamento } \\
\text { do intervalo QT }\end{array}$ & Grave & Farmacodinâmico & Razoável & 6 & $2,6 \%$ \\
\hline Enalapril + hidroclorotiazida & $\begin{array}{l}\text { Redução da pressão arterial } \\
\text { sistêmica }\end{array}$ & Moderada & Farmacodinâmico & Razoável & 6 & $2,6 \%$ \\
\hline Amitriptilina + difenidramanina & $\begin{array}{l}\text { Aumento dos efeitos anticolinérgicos } \\
\text { (por exemplo, xerostomia, retenção } \\
\text { urinária) }\end{array}$ & Moderada & Farmacodinâmico & Bom & 5 & $2,1 \%$ \\
\hline Atenolol + metformina & $\begin{array}{l}\text { Alterações glicêmicas. Redução dos } \\
\text { sintomas de hipoglicemia }\end{array}$ & & Farmacodinâmico & Bom & 5 & $2,1 \%$ \\
\hline Insulina + metformina & Aumento do risco de hipoglicemia & Moderada & Farmacodinâmico & Bom & 5 & $2,1 \%$ \\
\hline Enalapril + metformina & Aumento do risco de hipoglicemia & Moderada & Farmacodinâmico & Razoável & 4 & $1,7 \%$ \\
\hline Outras & & & & & 161 & $69,3 \%$ \\
\hline Total & & & & & 233 & $100 \%$ \\
\hline
\end{tabular}

*AAS: ácido acetilsalicílico.

principais complicaçóes desses eventos adversos incluem neutropenia, anemia e trombocitopenia ${ }^{19}$.

Pacientes oncológicos possuem múltiplos fatores para desenvolvimento de arritmias. Desse modo, o uso concomitante de fármacos que aumentam o risco de prolongamento do intervalo QT (por exemplo, leuprolide e trazodona ou venlafaxina; amitriptilina e ondansetrona) deve ser avaliado com cautela, tendo em vista o risco de eventos adversos, inclusive, fatais ${ }^{17,20}$.

A interação entre tramadol e ondansetrona foi frequente na nossa casuística e apresenta risco de induzir síndrome serotoninérgica. Portanto, recomenda-se monitorar os sinais clínicos de sua manifestação (por exemplo, ataxia, hiper-reflexia, tremores, agitação e outros $)^{6}$.

Salienta-se a alta frequência de fármacos com índice terapêutico estreito (por exemplo, anticonvulsivantes e anticoagulantes), envolvidos nas interaçóes encontradas, achado em consonância com investigaçóes prévias ${ }^{16,17}$. A fenitoína e outros anticonvulsivantes possuem interaçôes potenciais com antineoplásicos e, por esse motivo, podem causar descompensação de crises epiléticas, principalmente, no caso de acometimentos neoplásicos cerebrais. Assim, é preconizado o monitoramento sérico dos anticonvulsivantes e da efetividade dos antineoplásicos ${ }^{21}$.
Foi observado, em estudo comparativo envolvendo varfarina, que pacientes oncológicos apresentaram piores valores de Target Therapeutic Range, maior risco de sangramento e eventos tromboembólicos. Ademais, verificou-se associação positiva entre quimioterapia e desfechos desfavoráveis relacionados ao uso de anticoagulante. Recomenda-se, portanto, que o controle da Razão Normatizada Internacional seja realizado mais frequentemente durante o período de uso concomitante de varfarina com os antineoplásicos ${ }^{22}$. No presente estudo, foram identificadas interaçōes entre varfarina e cisplatina, fluoruracila e gencitabina.

Interaçôes medicamentosas que aumentam a carga anticolinérgica (amitriptilina e difenidramina) e medicamentos com alta atividade anticolinérgica devem ser evitados em idosos em razáo dos potenciais eventos adversos em nível central (sedação, confusão e declínio cognitivo) e em nível periférico (visão turva, constipação, tremores, retenção urinária e xerostomia ${ }^{23}$. Na oncologia, destaca-se o agravamento e/ou potencializaçáo do risco de mucosite (lesão na cavidade orogástrica) por causa da xerostomia induzida por fármacos. Tal lesão provoca eventos clínicos importantes como infecçôes oportunistas, dor, dificuldades para mastigação e deglutição $0^{24}$. 
A ocorrência de interação medicamentosa foi associada positivamente com número de problemas de saúde e com número de medicamentos; ou seja, a medida que aumenta número de medicamentos, há aumento no risco de interaçôes. Esse achado está em consonância com literatura consultada, na qual a polifarmácia apresenta-se como uma das principais variáveis associadas ao risco de interaçóes medicamentosas ${ }^{8-13}$. Hersh et.al ${ }^{25}$ referem que os fatores de risco para polifarmácia na população oncogeriátrica são o número de problemas de saúde (e de prescritores), hospitalização e prescrição para tratar eventos adversos a outros medicamentos. Os autores evidenciaram que as consequências da polifarmácia são eventos adversos, má adesão ao tratamento, interaçôes medicamentosas, fragilidade e aumento do risco de morbimortalidade.

Nesse contexto, medidas são necessárias para amenizar os riscos advindos das interaçóes medicamentosas, ainda que, em alguns casos, tais interaçôes sejam desejáveis. Autores sugerem revisão da farmacoterapia (incluindo fármacos antineoplásicos, terapias complementares e alternativas, bem como automedicação) com intuito de reduzir o número de medicamentos; identificação prévia de interaçôes potenciais e manejo clínico prospectivo adequado; ações interdisciplinares envolvendo profissionais de saúde de diversas áreas do conhecimento (farmacêuticos, geriatras, gerontólogos, oncologistas, entre outros) ${ }^{18,25}$.

Este estudo apresenta como limitação a realização em um único ambulatório, com inclusão apenas de idosos em quimioterapia parenteral e com funcionalidade relativamente preservada. Assim, não é possível extrapolar os dados encontrados para população idosa hospitalizada, frágil ou em uso de antineoplásicos orais.

Considerando a frequência de interaçóes fármaco-fármaco identificada nessa investigação, é válido destacar a importância de medidas interdisciplinares no cuidado ao paciente oncológico visando à reduçáo do risco de eventos adversos e à melhoria dos resultados terapêuticos. Para prestar cuidado fundamentado em evidências ao paciente com câncer, é importante investigar o impacto clínico das interaçôes na efetividade e na segurança do tratamento antineoplásico de idosos.

\section{CONCLUSÃO}

A frequência de interaçôes medicamentosas potenciais em idosos com câncer em terapia antineoplásica parenteral foi elevada, ainda que a proporção de interaçôes graves corresponda à, aproximadamente, metade do total de interaçóes. O número de problemas de saúde e o número de medicamentos apresentaram associação positiva com interaçôes medicamentosas.

\section{CONTRIBUIÇÕES}

Todos os autores participaram da concepção e delineamento do estudo; coleta, análise e interpretação dos dados; redação e revisão crítica do conteúdo; aprovação final da versão a ser publicada e se responsabilizam por todos os aspectos do trabalho.

\section{DECLARAÇÃO DE CONFLITO DE INTERESSES}

Nada a Declarar.

\section{REFERÊNCIAS}

1. Soares LC, Santana MG, Muniz RM. O fenômeno do câncer na vida de idosos. Ciência, Cuid e Saúde. 2010;9(4):660-7.

2. Pereira EEB, Santos NB, Sarges ESNF. Avaliação da capacidade funcional do paciente oncogeriátrico hospitalizado. Rev Pan-Amazônica Saúde. 2014;5(4):37-44.

3. Reis CM, Santos AG, Souza PJ, Reis AMM. Factors associated with the use of potentially inappropriate medications by older adults with cancer. J Geriatr Oncol. 2017;8(4):303-7.

4. Nightingale G, Pizzi LT, Barlow A, Barlow B, Jacisin T, McGuire $M$, et al. The prevalence of major drug-drug interactions in older adults with cancer and the role of clinical decision support software. J Geriatr Oncol; 2018.

5. National Comprehensive Cancer Network. The NCCN clinical practice guidelines in oncology senior adult oncology (version 2. 2017). 2017; [acesso em 2018 jun 01]. Disponível em: https://www.nccn.org/professionals/ physician_gls/default.aspx.

6. IBM Micromedex ${ }^{\circledR}$ Drug Interaction Checking[Internet]., Greenwood Village: Truven Health Analytics. [acesso em 2017 jun 10]. Disponível em: https://www. micromedexsolutions.com/home/dispatch/ssl/true.

7. Luz LL, Santiago LM, Silva JFS, Mattos IE. Psychometric properties of the Brazilian version of the Vulnerable Elders Survey-13 (VES-13). Cad Saúde Pública. 2015;31(3):507-15.

8. Puts MTE, Monette J, Girre V, Costa-Lima B, Wolfson $\mathrm{C}$, Batist $\mathrm{G}$, et al. Potential medication problems in older newly diagnosed cancer patients in Canada during cancer treatment: A prospective pilot cohort study. Drugs and Aging. 2010;27(7):559-72.

9. Girre V, Arkoub H, Puts MTE, Vantelon C, Blanchard F, Droz JP, et al. Potential drug interactions in elderly cancer patients. Crit Rev Oncol Hematol. 2011;78(3):220-6.

10. Popa MA, Wallace KJ, Brunello A, Extermann M. Potential Drug Interactions and Chemotoxicity in. older patients with cancer receiving chemotherapy. J Geriatric Oncol. 2015;5(3):307-14. 
11. Alkan A, Yasar A, Karci E, Köksoy EB,Ürün M, Senler FC, et al. Severe drug interactions and potentially inappropriate medication usage in elderly cancer patients. Support Care Cancer. 2017;25(1):229-36.

12. Ting YT, Yin TX, Si P, Chew L. Drug-related problems in elderly patients with cancer receiving outpatient chemotherapy. J Geriatr Oncol. 2015;6(4):280-7.

13. Rougé-Bugat ME, Bourgouin M, Gérard S, Lozano $S$, Brechemier D, Cestac P, et al. Drug prescription including interactions with anticancer treatments in the elderly: A global approach. J Nutr Heal Aging. 2017;1-6.

14. Raschi E, Piccinni C, Signoretta V, Lionello L, Bonezzi $S$, Delfino $M$, et al. Clinically important drug-drug interactions in poly-treated elderly outpatients: A campaign to improve appropriateness in general practice. Br J Clin Pharmacol. 2015;80(6):1411-20.

15. Georgy A, Neceskas J, Goodin S. Antiemetic care for patients with breast cancer: Focus on drug interactions and safety concerns. Am J Heal Pharm. 2007;64(21):2227-36.

16. Riechelmann RP, Del Giglio A. Drug interactions in oncology: How common are they? Ann Oncol. 2009;20(12):1907-12.

17. Van-Leeuwen RWF, Swart EL, Boven E, Boom FA, Schuitenmaker MG, Hugtenburg JG. Potential drug interactions in cancer therapy: A prevalence study using an advanced screening method. Ann Oncol. 2011;22(10):2334-41.

18. Lopez-Martin C, Garrido S, Alcaide-Garcia J, Faus FV. Role of clinical pharmacists to prevent drug interactions in cancer outpatients: a single-centre experience. Int J Clin Pharm. 2014;36(6):1251-9.

19. Hurria A, Browner IS, Cohen HJ, Denlinger CS, Shazo M, Extermann M, et al. Senior adult oncology: Clinical practice guidelines in oncology. J Natl Compr Cancer Netw. 2012;10(2):162-209.

20. Becker TK, Yeung SCJ. Drug-induced QT interval prolongation in cancer patients. Oncol Rev. 2010;4:223-32.

21. Yap KYL, Chui WK, Chan A. Drug interactions between chemotherapeutic regimens and antiepileptics. Clin Ther. 2008;30(8):1385-407.

22. Rose AJ, Sharman JP, Ozonoff A, Henault LE, Hylek EM. Effectiveness of warfarin among patients with cancer. J Gen Intern Med. 2007;22(7):997-1002.

23. Pfistermeister B, Tümena T, Gabmann KG, Maas R, Fromm MF. Anticholinergic burden and cognitive function in a large German cohort of hospitalized geriatric patients. PLoS One. 2017;12(2):1-13.

24. Holmes TSV, Santos MGC,Nóbrega DRM, Pereira JV, Gomes DQC, Pereira MSV. Fatores relacionados ao surgimento e gradação da mucosite oral radioinduzida. Rev Cubana Estomatol. 2014;51(1):71-9.
25. Hersh LR, Beldowski K, Hajjar ER. Polypharmacy in the Geriatric Oncology Population. Curr Oncol Rep. 2017;19(11):73. 